\title{
Ulceration of the entire small intestine accompanied by rash, hepatitis and pancytopenia
}

\author{
R.H.R. Park' ${ }^{\text {, P.R. Mills }}{ }^{1}$, B.J.Z. Danesh ${ }^{1}$, R.I.Russell ${ }^{1}$, D.H. Kennedy ${ }^{3}$, \\ C. McArdle ${ }^{2}$ and F.D. Lee ${ }^{4}$ \\ ${ }^{1}$ Gastroenterology Unit, ${ }^{2}$ University Department of Surgery and ${ }^{4}$ Department of Pathology, Royal Infirmary, \\ Glasgow and ${ }^{3}$ Clinical Department of Infectious Diseases, Ruchill Hospital, Glasgow, UK.
}

Summary: A case of acute mucosal ulceration of the entire small intestine accompanied by skin rash, hepatitis and marrow suppression is reported. Recovery was complicated by a severe protein losing enteropathy and small intestinal strictures and the patient died post-operatively of a pulmonary embolism. The aetiology remains unknown.

\section{Introduction}

Ulceration of the small intestine distal to the duodenum may be associated with Crohn's disease, idiopathic chronic ulcerative enteritis, polyarteritis, ischaemia, drugs or neoplasms. ${ }^{1,2}$ The ulceration is usually localized although multiple areas throughout the small intestine can be involved., Small bowel histology frequently shows transmural changes and many cases present with acute perforation. ${ }^{5}$ We report a case of fatal acute mucosal ulceration of the entire small intestine, which may have been drug related.

\section{Case report}

A 46 year old woman was admitted in May 1984 to an infectious diseases unit with a 5-day history of an influenza-like illness, widespread maculo-papular rash, nausea, vomiting and jaundice. On the day of admission she had developed generalized abdominal pain with watery, blood-stained diarrhoea. She had previously been well apart from low back pain of two months duration for which she had taken diclofenac sodium (Voltarol retard) for 3 weeks, followed by a 3 week course of suprofen (Suprol) and 2 weeks before admission had taken a combination of paracetamol and chlormezanone (Lobak).

On admission she was jaundiced, apyrexial and had a widespread maculo-papular rash. Apart from a tachycardia of $100 / \mathrm{min}$, cardiorespiratory examination was normal. She had slight abdominal tenderness. Initial results showed normal haemoglobin, white cell count, erythrocyte sedimentation rate, urea and elect-

Correspondence: R.H.R. Park, M.B., Ch.B., M.R.C.P., Gastroenterology Unit, The Royal Infirmary, Glasgow G31 2ER, UK.

Accepted: 26 July 1988 rolytes, serum bilirubin $49 \mu \mathrm{mol} / 1(2.9 \mathrm{mg} / \mathrm{dl})$, aspartate aminotransferase activity $930 \mathrm{IU} / \mathrm{l}$, alanine aminotransferase activity $1545 \mathrm{IU} / \mathrm{l}$, alkaline phosphatase activity $455 \mathrm{IU} / 1$ (laboratory reference range up to $92 \mathrm{IU} / \mathrm{l}$ ), total protein $69 \mathrm{~g} / \mathrm{l}$, albumin $46 \mathrm{~g} / \mathrm{l}$, gamma glutamyl transpeptidase activity 799 IU/l. Forty-eight hours later she became pancytopenic with a total white blood count of $1.6 \times 10^{9} / 1,31 \%$ neutrophils, haemoglobin $10.9 \mathrm{~g} / \mathrm{dl}$, platelets $110,000 \times$ $10^{9} / 1$. Coagulation screen was normal. Bone marrow aspirate showed a hypocellular marrow with maturation arrest of all cell lines. Within a few days of admission the pancytopenia, rash and abnormal liver function tests had all resolved. A liver biopsy was not performed in view of the clinical improvement. However, her diarrhoea persisted and she rapidly lost $5 \mathrm{~kg}$ in weight. On two occasions she passed string-like slough per rectum (Figure 1).

Initially an infective aetiology was suspected but stool investigations for the presence of salmonellae, shigellae, campylobacter, parasites, enteroviruses, Clostridium difficile and toxin, and serological tests for yersiniosis, amoebiasis, typhoid, hepatitis A, hepatitis B, Epstein-Barr virus,cytomegalovirus and parvovirus were all negative. Small bowel radiology demonstrated continuous mucosal oedema and ulceration with reduction in the bowel lumen from the third part of duodenum to the terminal ileum. The proximal colon appeared normal (Figure 2). A jejunal biopsy showed complete mucosal ulceration to the level of the muscularis mucosa with oedema of the submucosa and the mucosa replaced by granulation tissue intensely infiltrated by lymphocytes, plasma cells and eosinophils (Figure 3). Sigmoidoscopy rectal biopsy and upper gastrointestinal endoscopy with biopsy from the second part of duodenum were normal.

(C) The Fellowship of Postgraduate Medicine, 1989 


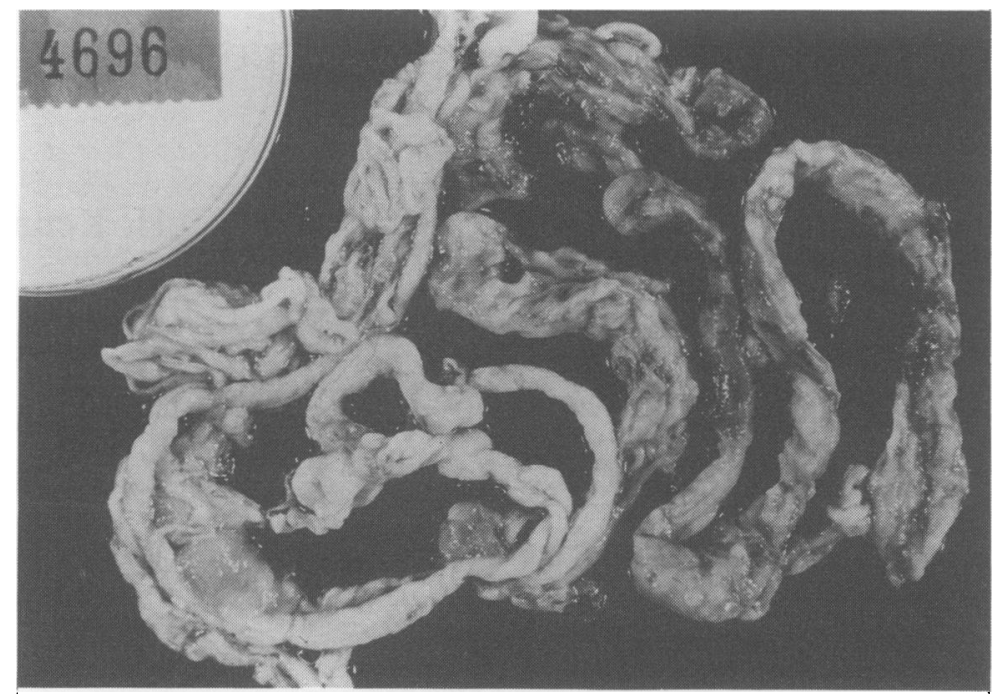

Figure 1 String like slough passed per rectum.

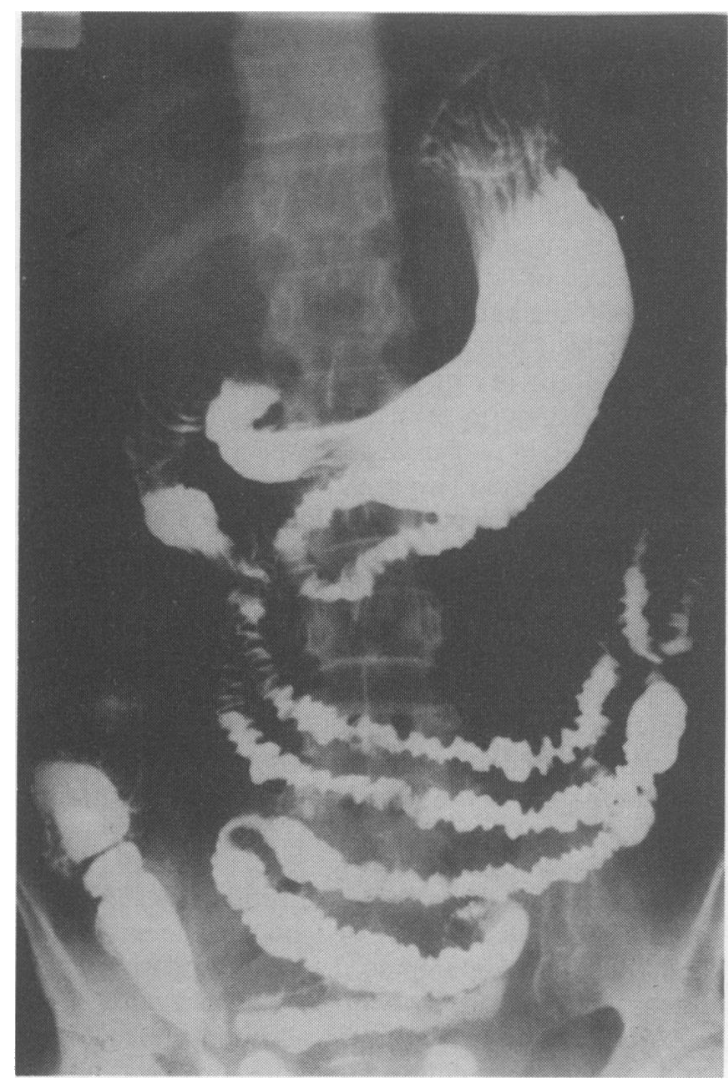

Figure 2 Barium meal and follow through.

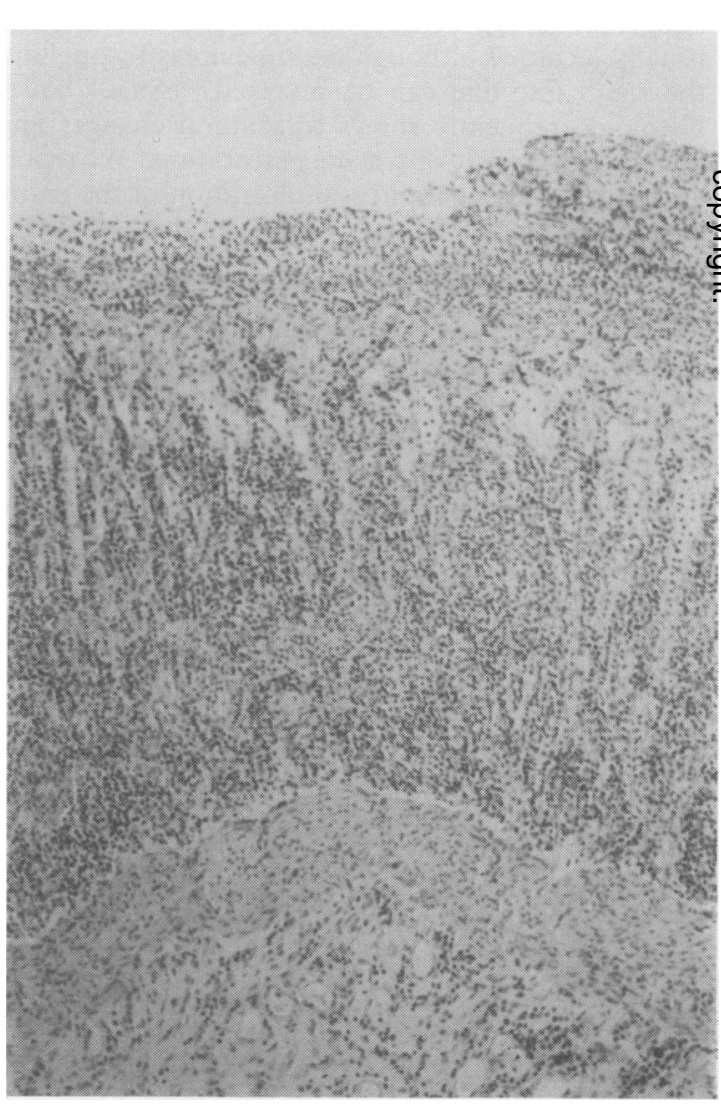

Figure 3 Jejunal biopsy (haematoxylin and eosin $\times 50$ ). 
By the fourth week, at the time of transfer, her serum albumin had dropped to $14 \mathrm{~g} / 1$ due to a severe protein losing enteropathy measured at $710 \mathrm{ml} /$ day (normal $<25 \mathrm{ml} /$ day by the ${ }^{51} \mathrm{CrCl}_{3}$ method). A 6-week course of intravenous nutrition with bowel rest, high dose steroids and albumin infusions failed to produce any clinical response. She subsequently developed bilious vomiting due to proximal small bowel strictures. In view of the obstructive symptoms and deteriorating clinical condition a laparotomy was performed at which the entire small intestine looked abnormal with slight serosal hyperaemia, absence of peristaltic activity and a thickened mesentery with enlarged lymph nodes. Approximately $60 \%$ of the small intestine from proximal jejunum to distal ileum was removed. The resected specimen showed continous ulceration throughout its length (Figure 4). On the luminal surface there was a confluent fibrinopurulent pseudo-membrane which was partially detached in places. No doubt this was the source of the string-like slough passed per rectum early on in the course of the disease. The histology was similar to the jejunal biopsy. In particular there was only minimal transmural inflammation, no granulomas in the bowel wall and no evidence of vascular or neoplastic disease. At the distal resection edge the villous architecture was almost normal and the inflammatory changes were relatively mild. In this distal area eosinophils were particularly numerous and there was also apoptotic cell damage.

Her clinical condition improved slightly but 7 weeks post-resection she died of a pulmonary embolus. Post-mortem examination failed to provide any additional information. Histology of the small intestine was unchanged. There were no significant abnormalities in the renal or hepatic histology.

\section{Discussion}

This case appears unique in several of its features. The acute onset of almost complete mucosal ulceration of the whole of the small bowel in combination with a skin rash, hepatitis and marrow suppression all suggest a toxic insult. Extensive investigations failed to reveal any infective agent and there was no clinical nor histological evidence of pre-existing enteropathy, inflammatory bowel disease or vascular insufficiency. The small bowel ulceration persisted and led to severe incapacity and multiple strictures while the other features settled rapidly. This clinical picture has not been described before and raises the possibility of an idiosyncratic drug reaction.

There is no doubt that exposure to non-steroidal anti-inflammatory agents took place. There is also pathological evidence to support the possibility of drug involvement including the apoptotic cell damage and the presence of numerous eosinophils in the distal ileum which had not undergone frank ulceration.,7 Unfortunately as the patient had taken three different drugs shortly before her presentation it is difficult to implicate one drug. However, the Committee on Safety of Medicines has received one report of enteritis (although not extensive small bowel ulceration), with diclofenac sodium, and many reports of various other gastrointestinal side effects with diclofenac sodium, suprofen, chlormezanone and paracetamol (Committee on Safety of Medicines - personal communications). The exact aetiology of this fatal case remains unknown. Although drug-related disease remains a conceivable cause, the low back pain may have indicated the onset of the disease process before the introduction of the drugs.

This case has been reported to the Committee on Safety of Medicines.

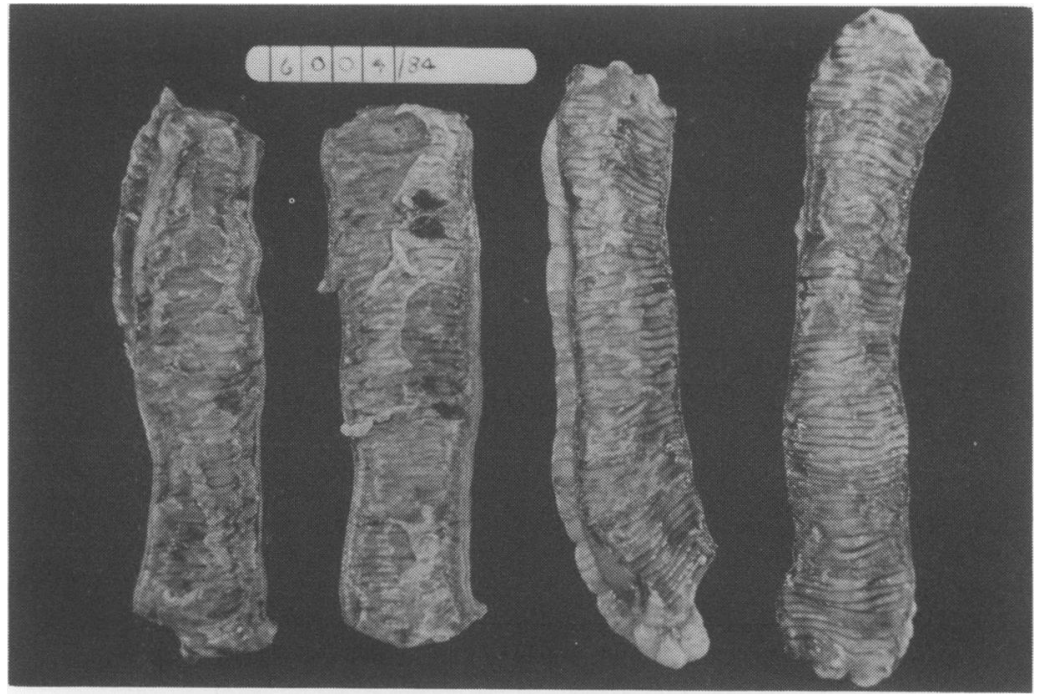

Figure 4 Resected small bowel. 


\section{References}

1. Jewell, D.P. Ulcerative enteritis. $\mathrm{Br}$ Med J 1983 287: 1740-1741.

2. Mills, P.R. Small intestine ulceration. Curr Opin Gastroenterol 1985, 1: 254-256.

3. Glynn, M.J., Pendower, J., Shousha, S. \& Parkins, R.A. Recurrent bleeding from idiopathic ulceration of small bowel. Br Med J 1983, 288: 975-976.

4. Thomas, W.E.G. \& Willamson, R. Idiopathic ulceration of the small bowel. Br Med J 1984, 288: 1835.

5. Davies, D.R. \& Brightmore, T. Idiopathic and drug induced ulceration of the small intestine. Br J Surg 1970, 57: $134-139$.
6. Rampton, D.S. Non-steroidal anti-inflammatory drugs and the lower gastrointestinal tract. Scand J Gastroenterol 1987, 22: 1-4.

7. Sommerville, K.W. \& Hawkey, C.J. Non-steroidal antiinflammatory agents and the gastrointestinal tract. Postgrad Med J 1986, 62: 23-28. 